\title{
NOTES
}

\section{Emulsion Polymerization of Chloroprene: The Mark-Houwink-Sakurada Equation for Linear Monodisperse and Branched Polychloroprene in a $\Theta$-Solvent}

\author{
Kenji Itoyama, Norihiro Shimizu*, and Shoya Matsuzawa* \\ Denka Techno-Research Co., Ltd., (1-3-D-6, Nakase, Chiba 260, Japan) \\ *Denki Kagaku Kogyo Co., Ltd., (4-1, Yurakucho 1-chome, \\ Chiyoda-ku, Tokyo 100, Japan)
}

(Received December 25, 1990)

\begin{abstract}
KEY WORDS Polychloroprene / Mark-Houwink-Sakurada Equation / $\Theta$-Solvent / Linear / Branch / Monodisperse / Molecular Weight Distribution / Emulsion Polymerization
\end{abstract}

The Mark-Houwink-Sakurada equation (MHS eq.) in a $\Theta$-solvent was already reported by Hanafusa et al. ${ }^{1}$ for polchloroprene. It is still unclear whether the relation could apply to linear and/or monodisperse polymer. That is an essential for analysing structure of polymers on the basis of dilute solution theory.

In polychloroprene, polymerized by radical reaction of diene monomer, there is some possibility of branching that may enlarge molecular weight distribution. So that it has been diffucult to establish the MHS eq. for linear monodisperse polymer.

In this paper, we firstly discuss the MHS eq. for linear monodisperse polychloroprene and secondarily examine the branching of polychloroprene.

\section{EXPERIMENTAL}

\section{Preparation of Sample}

The method of emulsion polymerization was the same as reported elsewhere. ${ }^{2}$ Emulsion polymerization of polychloroprene was carried out in the presence of a chain transfer reagent, $n$-dodecylmercaptan and lower (about 10\%) and higher $(95 \%)$ conversion polymers were prepared. Higher conversion polymer was fractionated by the method similar to that of Hanafusa et al., ${ }^{1}$ i.e., with benzene as a solvent and methanol as a non-solvent at $25^{\circ} \mathrm{C}$.

\section{Measurements}

The number-average molecular weight, $\bar{M}_{n}$, weight-average molecular weight, $\bar{M}_{w}$ and intrinsic viscosity, $[\eta]$ of polychloroprene in a $\Theta$-solvent (MEK, $25^{\circ} \mathrm{C}$ ) were measured using a Mechrolab 502 high speed membrane osmometer, a Shimadzu light scattering photometer and a capillary viscometer of the Ubberohde suspended level type, respectively. The method is identical to that performed by Hanafusa et al. ${ }^{1}$

\section{RESULTS AND DISCUSSION}

Investigation of the MHS eq. for Linear Monodisperse Polymer

$\bar{M}_{n}, \bar{M}_{w}$ and $[\eta]$ measured for all fractionated samples of both lower (about 10\%) and higher $(95 \%)$ conversion polychloroprene are summarized in Table I. The data of the first eleven rows in Table I are on lower conversion reported by Hanafusa et al., ${ }^{1}$ those of the 


\section{K. Itoyama, N. Shimizu, and S. Matsuzawa}

Table I. Molecular weight data o whole and fractionated samples of low and high conversion polymers

\begin{tabular}{|c|c|c|c|c|c|c|c|c|c|}
\hline Amount of DDM & Conv. & Sample & Whole or & {$[\eta]$} & $\bar{M}_{w}$ & $\bar{M}_{n}$ & $\bar{M}_{w} / \bar{M}_{n}$ & $K_{w}$ & $K_{n}$ \\
\hline$w t \%$ & $\%$ & No. & fract'd & & $\times 10^{-4}$ & $\times 10^{-4}$ & & $\times 10^{3}$ & $\times 10^{3}$ \\
\hline \multirow[t]{11}{*}{ - } & ca. 10 & $f-2$ & $\mathrm{~F}$ & 0.975 & 79.4 & - & - & 1.09 & - \\
\hline & & -3 & $F$ & 0.895 & 63.3 & 42.0 & 1.50 & 1.13 & 1.35 \\
\hline & & -4 & $\mathrm{~F}$ & 0.870 & 56.2 & 38.0 & 1.45 & 1.16 & 1.41 \\
\hline & & -6 & $\mathrm{~F}$ & 0.754 & 41.0 & 29.1 & 1.40 & 1.18 & 1.40 \\
\hline & & -7 & $\mathrm{~F}$ & 0.715 & 37.0 & 26.7 & 1.39 & 1.18 & 1.38 \\
\hline & & -8 & $\mathrm{~F}$ & 0.646 & 30.9 & 23.8 & 1.30 & 1.16 & 1.32 \\
\hline & & -0 & W & 0.714 & & & & & \\
\hline & & $F-2$ & $\mathrm{~F}$ & 0.571 & 24.1 & 19.8 & 1.22 & 1.16 & 1.28 \\
\hline & & -3 & $\mathrm{~F}$ & 0.500 & 19.2 & 15.6 & 1.23 & 1.14 & 1.27 \\
\hline & & -4 & $\mathrm{~F}$ & 0.430 & 15.3 & 11.9 & 1.29 & 1.10 & 1.25 \\
\hline & & -6 & $\mathrm{~F}$ & 0.274 & 5.6 & - & - & 1.16 & - \\
\hline 0.134 & 12.1 & & W & 0.808 & 48.5 & 23.9 & 2.03 & 1.16 & 1.65 \\
\hline 0.230 & 9.9 & & W & 0.575 & 24.3 & 12.9 & 1.88 & 1.17 & 1.60 \\
\hline 0.320 & 10.3 & & W & 0.474 & 17.7 & 8.6 & 2.06 & 1.13 & 1.62 \\
\hline \multirow[t]{8}{*}{0.500} & 95.0 & $f-1$ & $\mathrm{~F}$ & 0.755 & 463. & 35.9 & 12.9 & 0.35 & 1.26 \\
\hline & & -2 & $\mathrm{~F}$ & 0.639 & 70.7 & 32.1 & 2.20 & 0.76 & 1.13 \\
\hline & & -3 & $\mathrm{~F}$ & 0.576 & 38.9 & 25.6 & 1.50 & 0.92 & 1.14 \\
\hline & & -4 & $\mathrm{~F}$ & 0.509 & 25.3 & 19.4 & 1.30 & 1.01 & 1.16 \\
\hline & & -5 & $\mathrm{~F}$ & 0.443 & 17.7 & 15.8 & 1.12 & 1.05 & 1.11 \\
\hline & & -6 & $\mathrm{~F}$ & 0.395 & 12.6 & 11.8 & 1.07 & 1.11 & 1.15 \\
\hline & & -7 & $\mathrm{~F}$ & 0.347 & 10.8 & 9.1 & 1.19 & 1.06 & 1.15 \\
\hline & & -0 & W & 0.605 & & & & & \\
\hline
\end{tabular}

${ }^{\text {a }}$ Polym. temp, $40^{\circ} \mathrm{C}$.

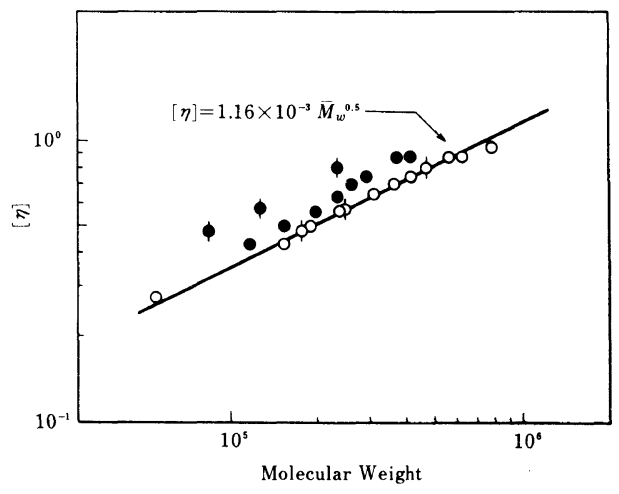

Figure 1a. Molecular weight $v s .[\eta]$ for low conversion samples. $0, \bar{M}_{n} ; \bigcirc, \bar{M}_{w} /$ fractionated samples. $\phi$, whole polymers.

second three rows are of whole polymer on lower (about 10\%) conversion which were polymerized with various amounts of chain

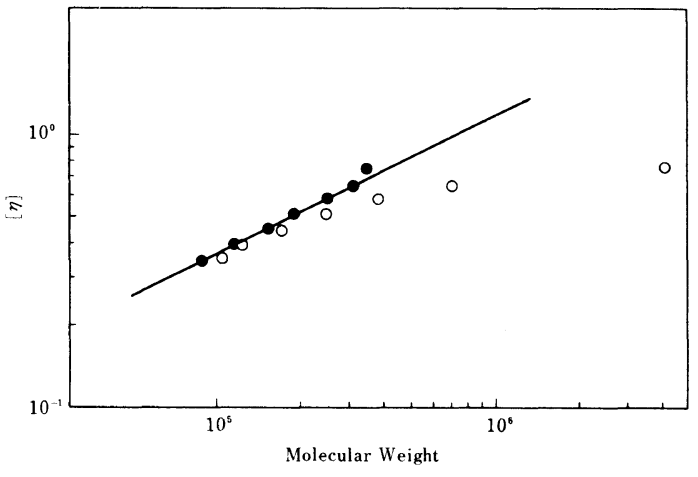

Figure 1b. Molecular weight $v s .[\eta]$ for high conversion fractionated samples., $\bar{M}_{n} ; \bigcirc, \bar{M}_{w}$.

transfer reagent, and those of the third eight rows are of fractionated ones on higher (95\%) conversion.

The relations between $[\eta]$ and molecular 


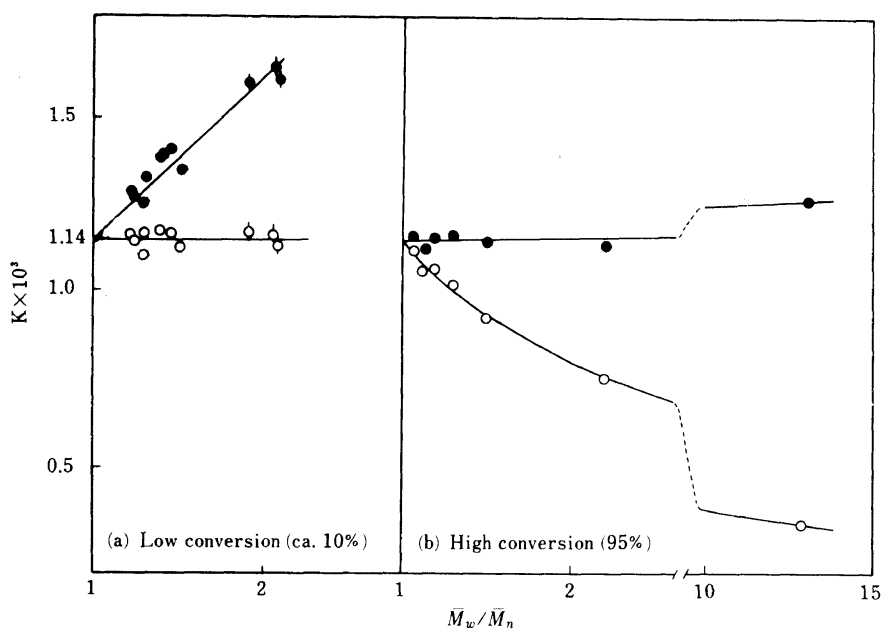

Figure 2. Plots of $K$ values $\left(K_{n}\right.$ and $\left.K_{w}\right)$ against $\bar{M}_{w} / \bar{M}_{n}$. (a) Low conversion (ca. 10\%). (b) High conversion $(95 \%)$.,$K_{n} ; \bigcirc, K_{w} /$ fractionated samples. $\phi$, $\phi$, whole polymers.

weight are shown in Figures $1 \mathrm{a}$ and $1 \mathrm{~b}$. In the lower conversion region the relationship: $[\eta]=1.16 \times 10^{-3} \times \bar{M}_{w}^{0.5}$, which has been reported by Hanafusa et al., ${ }^{1}$ still holds for both fractionated and whole polymers. On the other hand, in the higher conversion region, the experimental data deviate from the above relationship.

According to dilute solution theory, the relationship between $[\eta]$ and the molecular weight, $M$ of a given series of polymer homologs is described by the following MHS equation;

$$
[\eta]=K \cdot M^{a}
$$

where $K$ and $a$ are parameters on the temperature and the kinds of polymer and solvent. Since under the $\Theta$-condition (MEK, $25^{\circ} \mathrm{C}$ ), $a$ is equal to 0.5 , then $K$ can be calculated from experimental values of $[\eta]$ and $M$. If $K_{w}$ and $K_{n}$ are defined as parameters on $\bar{M}_{w}$ and $\bar{M}_{n}$, respectively;

$$
\begin{aligned}
K_{w} & =[\eta] / \bar{M}_{w}^{0.5} \\
K_{n} & =[\eta] / \bar{M}_{n}^{0.5}
\end{aligned}
$$

$K_{0}$ 's, i.e., $K$ 's of monodisperse polymers, are determined from the extrapolation of $K_{w}$ and
$K_{n}$ to $\bar{M}_{w} / \bar{M}_{n}=1$.

$K_{00}$, i.e., $K_{0}$ of a linear (without branching) monodisperse polymer, is obtained by extrapolating $K_{0}$ 's to zero conversion, because the branching density decreases to zero with decreasing conversion as confirmed in the previous paper. $^{2}$

$K_{w}$ and $K_{n}$ calculated from molecular weight and experimental $[\eta]$ are also tabulated in Table I. They are plotted against $\bar{M}_{w} / \bar{M}_{n}$ in Figures 2(a) and (b).

It is found from Figure 2 that extrapolating each of $K_{w}$ and $K_{n}$ to $\bar{M}_{w} / \bar{M}_{n}=1, K_{0}$ 's coincide wit each other irrespective of conversion. In addition, it has been confirmed that the polymer has essentially no branching at $10 \%$ conversion as mentioned later. Finally, we get

$$
[\eta]=1.14 \times 10^{-3} \cdot M^{0.5} \quad\left(\mathrm{dl} \mathrm{g}^{-1}\right)
$$

for linear monodisperse polychloroprene.

\section{Effect of Branching}

For evaluation of the long chain branching (LCB), based on the works by Zimm and Stockmayer, ${ }^{3}$ Kilb, ${ }^{4}$ and Kurata et al., ${ }^{5}$ Coleman and Fuller ${ }^{6}$ derived the following equation for randomly branched monodisperse polymer with tetrafunctional points: 
Table II. Branching parameters of fractionated samples of high conversion polymer

\begin{tabular}{|c|c|c|c|c|}
\hline \multirow{2}{*}{$\begin{array}{c}{[\text { Conv.] }} \\
\text { Sample No. }\end{array}$} & \multicolumn{2}{|c|}{$\begin{array}{l}\text { MWD: Exponential } \\
\text { type }\end{array}$} & \multicolumn{2}{|c|}{$\begin{array}{l}\text { MWD: Log-normal } \\
\text { type }\end{array}$} \\
\hline & $g$ & $\lambda \times 10^{7}$ & $g$ & $\lambda \times 10^{7}$ \\
\hline \multicolumn{5}{|l|}{$[95 \%]$} \\
\hline$f-1$ & 0.164 & 170. & 0.232 & 80.9 \\
\hline-2 & 0.555 & 64.0 & 0.582 & 55.5 \\
\hline-3 & 0.735 & 43.5 & 0.744 & 41.2 \\
\hline-4 & 0.835 & 34.0 & 0.840 & 32.7 \\
\hline-5 & 0.872 & 35.2 & 0.872 & 35.2 \\
\hline-6 & 0.947 & 18.0 & 0.947 & 18.0 \\
\hline-7 & 0.882 & 51.7 & 0.887 & 49.6 \\
\hline
\end{tabular}

$$
[\eta]_{0 \mathrm{~b}}=g^{0.6} \cdot K_{00} M^{0.5}
$$

where

$$
\begin{gathered}
{[\eta]_{0 \mathrm{~b}}=g^{0.6}[\eta]_{01}} \\
g=\left[\left(1+\frac{\lambda M}{6}\right)^{1 / 2}+\frac{4 \lambda M}{3 \pi}\right]^{-1 / 2}
\end{gathered}
$$

$\lambda$ is the number of branch points per unit molecular weight, $M$ is the molecular weight, and $g$ is the ratio of radius of gyration between branched and linear polymers. Subscripts 0 , 1 and $b$ designate $\Theta$-condition, linear and branched.

From the experimental data of higher conversion $(95 \%)$ in Table I, we obtained the branching parameter $\lambda$. For the convenience of the comparison of molecular weight distribution (MWD), we calculated on the assumption of both types of distribution. On the caluculation process, it is necessary to know $g$-value. In this work, we made correction of $[\eta]_{\mathrm{ob}}$ for molecular distribution by using correction factors, given by Kurata and Tsunashima. ${ }^{7}$ We also used $K_{00}=1.14 \times 10^{-3}$ similar to that obtained above. Results are tabulated in Table II. The effect of MWD is small except for $\mathrm{f}-1$ that has a quite large value of $\bar{M}_{\mathrm{w}} / \bar{M}_{n}$. It becomes clear that in the higher conversion there are cosiderable amount of branching.

In the previous paper, ${ }^{2}$ we discussed the crosslinking of polymers fromed in the absence of chain transfer reagent, and obtained "standard branching", i.e., crosslinking density without the effect of chain transfer reagent. This corresponds to $\lambda=7.2 \times 10^{-7}$ at $10 \%$ conversion (that denotes an essetially linear polymer as discussed above), and $\lambda=180 \times 10^{-7}$ at $95 \%$ conversion. Compared with the latter, the experimental data are of much lower values. These results suggest that chain transfer reagent performs not only a control of molecular weight but also an inhibition of the branching.

Acknowledgments. We would like to express our sincere thanks to Denki Kagaku Kogyo Co., Ltd., for supporting this study and Professor Riichiro Chûjô of Tokyo Institute of Technology for his helpful discussions.

\section{REFERENCES}

1. K. Hanafusa, A. Teramoto, and H. Fujita, J. Phys, Chem., 70, 4004 (1966).

2. K. Itoyama, N. Hirashima, J. Hirano, and T. Kadowaki, Polym. J., 23, 859 (1991).

3. B. H. Zimm and W. H. Stockmayer, J. Chem. Phys., 17, 1301 (1949).

4. B. H. Zimm and R. W. Kilb, J. Polym. Sci., 37, 19 (1959).

5. M. Kurata, M. Abe, M. Iwama, and M. Matsushima, Polym. J., 6, 729, 739 (1972).

6. M. M. Coleman and R. E. Fuller, J. Macromol. Sci. -Phys., B11, 419 (1975).

7. M. Kurata and Y. Tsunashima, "Polymer Handbook," Vol. VII, 3rd ed, John Wily \& Sons, New York, N. Y., 1989, p1-4. 